“ (C) 2016 IEEE. Personal use of this material is permitted. Permission from IEEE must be obtained for all other uses, in any current or future media, including

reprinting/republishing this material for advertising or promotional purposes, creating new collective works, for resale or redistribution to servers or lists, or reuse of any copyrighted component of this work in other works." 


\title{
Activating Protein Phosphatase 2A (PP2A) Enhances Tristetraprolin (TTP) Anti-Inflammatory Function in A549 Lung Epithelial Cells
}

\author{
Md. Mostafizur Rahman ${ }^{1}$, Nowshin N. Rumzhum ${ }^{1}$, Philip M. Hansbro ${ }^{2}$, Jonathan C. Morris ${ }^{3}$, \\ Andrew R. Clark ${ }^{4}$, Nicole M. Verrills ${ }^{5}$ and Alaina J. Ammit ${ }^{1}$ \\ ${ }^{1}$ Faculty of Pharmacy, University of Sydney, NSW 2006 Australia \\ ${ }^{2}$ Priority Research Centre for Respiratory Diseases, \\ Hunter Medical Research Institute and the University of Newcastle, NSW 2308 Australia \\ ${ }^{3}$ School of Chemistry, University of NSW, NSW 2052 Australia \\ ${ }^{4}$ Centre for Translational Inflammation Research, School of Immunity and Infection, University \\ of Birmingham, Edgbaston B15 2TT United Kingdom \\ ${ }^{5}$ School of Biomedical Sciences and Pharmacy, Faculty of Health and Medicine, University of \\ Newcastle, NSW 2308 Australia
}

Corresponding author:

\section{Phone:}

Fax:

E-mail:

Running title:

\author{
Alaina J. Ammit Ph.D. \\ Faculty of Pharmacy \\ University of Sydney \\ NSW 2006 Australia \\ +61293516099 \\ +61293514391 \\ alaina.ammit@sydney.edu.au
}

PP2A enhances TTP anti-inflammatory function 


\begin{abstract}
Chronic respiratory diseases are driven by inflammation, but some clinical conditions (severe asthma, COPD) are refractory to conventional anti-inflammatory therapies. Thus, novel antiinflammatory strategies are necessary. The mRNA destabilizing protein, tristetraprolin (TTP), is an anti-inflammatory molecule that functions to induce mRNA decay of cytokines that drive pathogenesis of respiratory disorders. TTP is regulated by phosphorylation and protein phosphatase 2A (PP2A) is responsible for dephosphorylating (and hence activating) TTP, amongst other targets. PP2A is activated by small molecules, FTY720 and AAL(S), and in this study we examine whether these compounds repress cytokine production in a cellular model of airway inflammation using A549 lung epithelial cells stimulated with tumor necrosis factor $\alpha$ (TNF $\alpha$ ) in vitro. PP2A activators significantly increase TNF $\alpha$-induced PP2A activity and inhibit mRNA expression and protein secretion of interleukin 8 (IL-8) and IL-6; two key proinflammatory cytokines implicated in respiratory disease and TTP targets. The effect of PP2A activators is not via an increase in TNF $\alpha$-induced TTP mRNA expression; instead we demonstrate a link between PP2A activation and TTP anti-inflammatory function by showing that specific knockdown of TTP with siRNA reversed the repression of TNF $\alpha$-induced IL-8 and IL-6 mRNA expression and protein secretion by FTY720. Therefore we propose that PP2A activators affect the dynamic equilibrium regulating TTP; shifting the equilibrium from phosphorylated (inactive) towards unphosphorylated (active) but unstable TTP. PP2A activators boost the anti-inflammatory function of TTP and have implications for future pharmacotherapeutic strategies to combat inflammation in respiratory disease.
\end{abstract}

Keywords: protein phosphatase 2A, tristetraprolin, TNF $\alpha$, inflammation, asthma 


\section{Introduction}

The prevalence of chronic respiratory disease is on the increase. Pathogenesis and disease progression are driven by inflammation, but in many clinical situations (severe asthma, infectious exacerbation, COPD), we do not have effective anti-inflammatory treatments [1-3]. Thus, alternative anti-inflammatory strategies are urgently needed. This is the potential of tristetraprolin (TTP).

TTP is an mRNA destabilizing molecule that acts at the post-transcriptional level to inhibit the expression of many pro-inflammatory cytokines responsible for respiratory disease pathogenesis (reviewed in [4]). TTP is regulated by phosphorylation at a number of levels; gene expression, mRNA and protein stability is p38 MAPK-dependent $[5,6]$ and its anti-inflammatory action is controlled by the phosphorylation status of TTP protein at S52 and S178 [5-7]. There is a dynamic equilibrium between unphosphorylated and phosphorylated TTP. In the unphosphorylated state, TTP is active and can induce mRNA decay however; this form of TTP is also subject to degradation by the ubiquitin-proteasome system [6, 8]. When TTP is phosphorylated, the protein is stabilized [8-10], but this phosphorylated form of TTP is unable to cause mRNA decay, and therefore is inactive [7].

When active, TTP has a substantial repressive impact on airway inflammation [6]. However, under respiratory disease conditions, although TTP is present, it is likely held in a dormant, inactive form due to the high levels of p38 MAPK phosphorylation [11]. Therefore developing a strategy to activate TTP under inflammatory conditions could be therapeutically useful. This dynamic equilibrium provides us with important points of regulatory control that we propose to exploit to enhance TTP function. However, blocking p38 MAPK with inhibitors in an attempt to promote greater amounts of unphosphorylated (active) TTP doesn't work because TTP is a p38 
MAPK-responsive gene and this will result in less TTP mRNA expression [5, 12]. A better approach is to allow TTP to be expressed and then activate its mRNA destabilizing function. This is the promise of protein phosphatase 2A (PP2A), a serine/threonine phosphatase responsible for dephosphorylation of TTP at S52 and S178 [13]; amongst other molecules [1416]. PP2A is a ubiquitously expressed enzyme and in a recent publication [17] we demonstrated the important regulatory role exerted by PP2A under basal (unstimulated conditions) in the A549 lung epithelial cell line. When PP2A basal enzymatic activity was removed, i.e. with the PP2A inhibitor okadaic acid or via specific knockdown of PP2A-C with siRNA, TTP was hyperphosphorylated and rendered non-functional [17]. Hence, proinflammatory cytokine production ensued. Therefore it follows that if TTP is switched off when PP2A activity is reduced, the corollary of these results is that TTP will be switched on by PP2A activators. This was the case under unstimulated conditions [17], but whether PP2A activators can switch TTP on and repress cytokine production under inflammatory conditions mimicked in vitro was currently unknown.

We address this herein by utilizing a cellular model of airway inflammation using A549 lung epithelial cells [17-19] stimulated with tumor necrosis factor $\alpha$ (TNF $\alpha)$ to mimic proinflammatory conditions in vitro $[17,18]$. We measure the impact on two key TTP targets implicated in respiratory disease, interleukin 8 (IL-8) and 6 (IL-6), as a functional outcome. We examine the ability of the sphingosine analog, FTY720, and the chiral analog of FTY720 devoid of sphingosine 1-phosphate (S1P) agonism, $\mathrm{AAL}(\mathrm{S})$ [17, 20-22] to activate PP2A and repress TNF $\alpha$-induced cytokine production in a TTP- dependent manner. Thus, in this study we address the hypothesis that PP2A activators can switch TTP on and repress cytokine production in an in vitro model of airway inflammation, and show for the first time that the PP2A activators FTY720 
and AAL(S) repress TNFa-induced IL-8 and IL-6 mRNA expression and protein secretion in a TTP-dependent manner. 


\section{Materials and Methods}

\subsection{Chemicals}

FTY720 was purchased from the Cayman Chemical Company (Ann Arbor, MI) and AAL(S) (2amino-4-(4-heptyloxyphenyl)-2-methylbutanol) was synthesized [17, 20-22]. TNF $\alpha$ is from R\&D Systems (Minneapolis, MN). Unless otherwise specified, all chemicals used in this study were purchased from Sigma-Aldrich (St. Louis, MO).

\subsection{Cell culture}

The human alveolar epithelial cell line (A549) was cultured in Ham's F-12K (Kaighn's) medium (Invitrogen, Carlsbad, CA) supplemented with penicillin (100 U/ml), streptomycin $(100 \mu \mathrm{g} / \mathrm{ml})$, and fetal calf serum $(10 \%)$ [17, 18]. All experiments were performed after an overnight serumstarvation period (14-16 h) in Ham's F-12K supplemented with sterile BSA (0.1\%) and stimulated with $4 \mathrm{ng} / \mathrm{ml} \mathrm{TNF} \alpha$ in accordance with conditions originally reported by Cornell et al. [18]. A minimum of three experimental replicates performed on separate days were used for each experiment.

\subsection{PP2A activity assay}

PP2A activity was determined using the PP2A immunoprecipitation phosphatase assay kit (Merck Millipore, Darmstadt, Germany) according to the manufacturer's instructions.

\subsection{Real-time RT-PCR}

Total RNA was extracted using the RNeasy Mini Kit (Qiagen Australia, Doncaster, VIC, Australia) and reverse transcription performed by using the RevertAid First strand cDNA 
Synthesis kit (Fermentas Life Sciences, Hanover, MD) according to the manufacturer's protocol. IL-8, IL-6 and TTP mRNA levels were measured using real-time RT-PCR on an ABI Prism 7500 (Applied Biosystems, Foster City, CA) with IL-8 (Hs00174103_m1), IL-6 (Hs00174131_m1) and TTP (Zfp36, Hs00185658_m1) TaqMan gene expression assays and the eukaryotic 18S rRNA endogenous control probe (Applied Biosystems) subjected to the following cycle parameters: $50^{\circ} \mathrm{C}$ for $2 \mathrm{~min}, 1 \mathrm{cycle} ; 95^{\circ} \mathrm{C}$ for $10 \mathrm{~min}, 1$ cycle; $95^{\circ} \mathrm{C}$ for $15 \mathrm{~s}$, $60^{\circ} \mathrm{C}$ for 1 min, 40 cycles and mRNA expression (fold increase) quantified by delta delta $\mathrm{Ct}$ calculations.

\subsection{ELISA}

IL-8 and IL-6 ELISAs were performed according to the manufacturer's instructions (BD Biosciences Pharmingen, San Diego, CA).

\subsection{Western blotting}

TTP was measured by Western blotting using rabbit antisera against TTP (Sak21) [5]. Detection of $\alpha$-tubulin was used as the loading control (mouse monoclonal $\operatorname{IgG}_{1}$, DM1A: Santa Cruz Biotechnology, Santa Cruz, CA). p38 MAPK was detected using rabbit monoclonal or polyclonal antibodies against phosphorylated $\left(\mathrm{Thr}^{180} / \mathrm{Tyr}^{182}\right)$ and total p38 MAPK (Cell Signaling Technology, Danvers, MA). Primary antibodies were detected with goat anti-rabbit and anti-mouse HRP-conjugated secondary antibodies (Cell Signaling Technology, Danvers, MA) and visualized by enhanced chemiluminescence (PerkinElmer, Wellesley, MA).

\section{7 siRNA}


A549 cells $\left(5 \times 10^{5}\right.$ cells/well $)$ were transfected with siRNA against TTP, or scrambled control, by reverse transfection with RNAiMAX according to the manufacturer's protocols (Invitrogen, NY, USA). Untransfected controls were also performed. Specifically, for each well of 6-well plates, $800 \mathrm{ng}$ of ON-Target plus Control Non-targeting siRNA (scrambled control) or ON-target plus SMART pool Human TTP siRNA (aka Zfp36: both from Dharmacon, Thermo-Fisher Scientific, Waltham, MA) was diluted in $500 \mu \mathrm{L}$ of Opti-MEM Reduced Serum Media (Invitrogen). This was followed by the addition of $5 \mu \mathrm{L}$ of RNAiMAX reagent (Invitrogen) into each well and incubation at room temperature for $20 \mathrm{~min}$. After transfection, cells were cultured for $24 \mathrm{~h}$ in media without antibiotics, and then growth-arrested for $16 \mathrm{~h}$ in Ham's F-12K supplemented with $0.1 \%$ BSA, supplemented with penicillin $(100 \mathrm{U} / \mathrm{ml})$, streptomycin $(100$ $\mu \mathrm{g} / \mathrm{ml})$, before stimulation with $\mathrm{TNF} \alpha(4 \mathrm{ng} / \mathrm{ml})$.

\subsection{Statistical analysis}

Statistical analysis was performed using either the Student's unpaired $t$ test, one-way or two-way ANOVA followed by Bonferroni's post-test. $P$ values $<0.05$ were sufficient to reject the null hypothesis for all analyses. Data are mean+SEM of $n \geq 3$ independent replicates. 


\section{Results}

\subsection{PP2A activators, FTY720 and AAL(S), enhance TNFa-induced PP2A phosphatase activity in A549 cells}

PP2A is a ubiquitous enzyme that exerts basal phosphatase activity. In confirmation of our earlier study [17], we show in Figure 1 that this basal activity can be significantly increased by stimulation for $1 \mathrm{~h}$ with TNF $\alpha(P<0.05)$. We then examined the impact of two PP2A activators: 1) the classical PP2A activator - FTY720; 2) the novel PP2A activator and chiral analog of FY720 devoid of S1P agonism - AAL(S). Notably, both PP2A activators can significantly augment TNF $\alpha$-induced PP2A activity (Figures 1A \& 1B: $P<0.05$ ), but are without effect when added to unstimulated cells.

3.2 PP2A activators, FTY720 and AAL(S), significantly repress TNF $\alpha$-induced IL-8 and IL-6 mRNA expression and protein secretion

We then wished to examine the impact of PP2A activation with FTY720 and AAL(S) on TNF $\alpha$ induced expression kinetics of two TTP targets - IL-8 (Figure 2) and IL-6 (Figure 3). As shown in Figure 2, TNFo significantly increased IL-8 mRNA expression (Figures 2A \& 2C) and protein secretion (Figures 2B \& 2D) from A549 cells $(P<0.05)$. Significant levels of IL-8 mRNA expression was first observed at $1 \mathrm{~h}$ and remained elevated throughout the $24 \mathrm{~h}$ period $(P<0.05)$. Protein production ensued with significantly elevated levels of IL-8 secretion detected from $4 \mathrm{~h}$ $(P<0.05)$. Notably, TNF $\alpha$-induced cytokine production could be repressed by pretreatment with PP2A activators. FTY720 significantly repressed TNF $\alpha$-induced IL-8 mRNA expression (Figure 2A) and cytokine secretion (Figure 2B) $(P<0.05)$. IL-8 mRNA expression (Figure 2C) and cytokine secretion (Figure 2D) was similarly repressed by pretreatment with AAL(S). To extend 
these observations, we also examined the impact of PP2A activators on another proinflammatory cytokine implicated in chronic respiratory disease - IL-6 - and results shown in Figure 3 confirm that PP2A activators significantly repress TNFo-induced IL-6 mRNA expression (Figures 3A \& 3C) and protein secretion (Figures 3B \& 3D) from A549 cells $(P<0.05)$. Notably, FTY720 and AAL(S) alone do not induce cytokine mRNA expression and cytokine secretion from A549 cells.

\section{3 Specific knockdown of TTP with siRNA increases TNF $\alpha$-induced IL-8 and IL-6 mRNA expression and protein secretion}

So far we have shown that PP2A activators - FTY720 and AAL(S) - increase PP2A activity and significantly inhibit TNF $\alpha$-induced IL-8 and IL-6 mRNA expression and cytokine secretion (Figures 1-3). We hypothesize that PP2A activators mediate their anti-inflammatory effect in a TTP-dependent manner. Towards testing this link, we first needed to show the impact of TTP on cytokine production under inflammatory conditions. To do this, we transiently transfected A549 cells with siRNA against TTP and stimulated cells with TNF $\alpha$, compared to relevant controls (Figure 4). As expected, TNF $\alpha$ increased TTP mRNA (Figure 1A: $P<0.05$ ) and TTP protein expression (Figure 1B). As shown in Figure 4A, siRNA against TTP significantly repressed TNF $\alpha$-induced TTP mRNA expression $(P<0.05)$. TNF $\alpha$-induced TTP protein production was similarly knocked down by siRNA against TTP, as demonstrated by Western blot (Figure 4B). Importantly, when TTP was reduced by siRNA, there was a corresponding increase in IL-8 and IL-6 mRNA expression (Figures 4C \& 4E) and cytokine secretion (Figures 4D \& 4F) from A549 cells $(P<0.05)$. These results demonstrate that TTP represses cytokine production in airway inflammatory conditions modeled in vitro. 


\subsection{PP2A activators do not enhance TTP mRNA expression; rather they modify TTP's dynamic equilibrium}

We next sought to examine the effect of PP2A activators on the temporal regulation of TTP mRNA expression. As shown in Figure 5A, TNF $\alpha$ significantly increased TTP mRNA expression with a peak at $1 \mathrm{~h}(P<0.05)$; however, this was unaffected by pretreatment with FTY720. Pretreatment with AAL(S) was similarly without effect on TNF $\alpha$-induced TTP mRNA expression (Figure 5B). We then examined the effect of PP2A activators on TTP protein levels. We initially established the temporal kinetics of TTP protein upregulation induced by TNF $\alpha$. TTP's anti-inflammatory function is controlled by phosphorylation and there is a dynamic equilibrium between unphosphorylated/active and phosphorylated/active TTP. As shown in Figure 5C, and in confirmation of earlier studies in A549 [17, 19] and other cell types [5, 12], the rabbit antisera Sak21 detects different phospho-forms of TTP; the immunoreactive bands at higher molecular weight indicate phosphorylated TTP (inactive), while lower bands are unphosphorylated (active). TTP is an immediate-early gene [23] and can be detected at $1 \mathrm{~h}$ in both phosphorylated and unphosphorylated forms. By $2 \mathrm{~h}$, phosphorylated TTP predominates and reduces over time (Figure 5C). In order to examine the effect of PP2A activators on these phospho-forms of TTP we focused in on the $1 \mathrm{~h}$ (Figure 5D) and $2 \mathrm{~h}$ time points (Figure 5E). As demonstrated by Western blotting and quantitated by densitometry, PP2A activators reduced the amount of TTP protein upregulated in response to TNF $\alpha$. This is seemingly counterintuitive, but we offer an explanation that is supported by recent in vivo studies $[6,24]$. We propose that PP2A activators affect TTP's dynamic equilibrium; shifting the equilibrium from phosphorylated (inactive) towards unphosphorylated (active) TTP. Because the phosphorylation of TTP both 
inactivates TTP and protects it from proteasome-mediated degradation, we predicted that PP2A agonists that promoted TTP dephosphorylation would decrease total TTP protein levels. This was exactly the outcome observed in Figures 5D \& 5E. To demonstrate that FTY720 and AAL(S) had no effect on another PP2A target, such as the phosphoprotein responsible for TNF $\alpha$-induced IL-8 and IL-6 production - p38 MAPK [17, 25-27] - we examined whether PP2A activators affected TNF $\alpha$-induced p38 MAPK phosphorylation. As shown in Figure 5F, PP2A activators had no effect on p38 MAPK activity.

\subsection{Specific knockdown of TTP with siRNA reverses repression of TNF $\alpha$-induced IL-8 and IL-6 mRNA expression and protein secretion by the PP2A activator FTY720}

Finally, we demonstrated a link between PP2A activation and TTP-mediated cytokine repression. We examined whether FTY720-mediated repression was TTP-dependent by knocking-down TTP with siRNA and observing the resultant effect on cytokine production. Figure 6A confirms that siRNA against TTP reduces TTP mRNA expression. In confirmation of earlier data, FTY720 significantly repressed TNF $\alpha$-induced IL-8 mRNA expression (Figure 6B) and protein secretion (Figure 6C) $(P<0.05)$. IL-6 mRNA expression and protein secretion was also significantly repressed by FTY720 (Figures 6D \& 6E: $P<0.05$ ). Notably, specific knockdown of TTP with siRNA reverses repression of IL-8 and IL-6 mRNA expression and protein secretion by FTY720 (Figures 6B-6E). These data support our hypothesis that PP2A activators mediate their anti-inflammatory effect in a TTP-dependent manner. 


\section{Discussion}

PP2A is a tri-molecular holoenzyme responsible for dephosphorylating (and hence activating) TTP, amongst many other targets. Small molecule PP2A activators have been developed and in this study we use two key examples - FTY720 and AAL(S) - to show that PP2A activators can enhance TTP's anti-inflammatory function to inhibit cytokine production in a cellular model of airway inflammation in vitro.

PP2A is best known for its role as a tumour suppressor responsible for the regulation of a range of cellular signalling pathways involved in proliferation, survival and migration in cancer [28]. Notably, PP2A expression/activity is reduced in a number of cancers [29, 30]. Thus, restoration of PP2A tumour-suppression activity by PP2A activators has emerged as a novel therapy for treating cancer. Excitingly, the knowledge gained from understanding of PP2A in cancer has propelled research into its role and function in respiratory disease. PP2A levels (and therefore activity) were reduced in mouse models of allergen- and rhinovirus-induced asthma [21] and could be reinstated pharmacologically with $\mathrm{AAL}(\mathrm{S})$ to repress hallmark features of airway disease [21, 22]. Notably, defects in PP2A cause corticosteroid insensitivity in severe asthma [31]. Reduced PP2A activity induces corticosteroid resistance and this can be reversed by the long-acting $\beta_{2}$-agonist formoterol [32]. Cornell et al. [18] showed that blocking PP2A robustly increased IL-8 expression in A549 cell via post-transcriptional mRNA stabilization; although TTP was implicated, it was not directly examined. With these studies, we and others have underscored the importance of investigations into the regulation and pharmacological modulation of PP2A in airway inflammation. But PP2A dephosphorylates a number of kinases that drive inflammatory pathways [14-16]; whether TTP was involved remained an open question. 
We address this herein. PP2A is the phosphatase responsible for TTP dephosphorylation [13]. Our earlier studies have shown the impact of PP2A inhibitors on TTP. Brook et al. [8] used a non-specific PP2A inhibitor calyculin A and demonstrated accumulation of the hyperphosphorylated in active form of TTP. More recently [17], we showed that when PP2A is reduced (either by a non-specific pharmacological inhibitor okadaic acid or more specifically with siRNA against PP2A-C) TTP is hyperphosphorylated and rendered inactive. Under these conditions, the homeostatic restraint that the ubiquitous enzyme PP2A exerts under basal conditions is lost and therefore cytokine secretion ensues. In the current study we build upon these observations and exploit the capacity of known PP2A activators to enhance PP2A phosphatase activity to effectively shift the equilibrium from phosphorylated/inactive towards unphosphorylated/active TTP.

A number of small molecules have been reported to activate PP2A [28]. To date, the best known of these is the sphingosine analog FTY720 (fingolimod; Gilenya (Novartis)). However FTY720 has other targets. Most notably, FTY720 is also a functional antagonist of the S1P pathway. However, in this study we assert that FTY720's repressive actions are primarily due to PP2A activation. This is demonstrated by the lack of effect of FTY720 on cytokine expression. For FTY720 to inhibit the S1P pathway, it must first be taken up by the cells, become phosphorylated intracellularly by sphingosine kinase 2 to FTY720-P. FTY720-P must then be released from cells to act back on $\mathrm{S} 1 \mathrm{P}$ receptors (S1PR1, 3, 4, 5, but not S1PR2), internalizing the receptors and preventing them from being activated again by native ligand S1P (functional antagonism). For this reason, we suggest that if FTY720 was acting via S1PR-mediated pathways, it should induce cytokine production as a necessary part of its receptor-mediated interaction and consequent S1PR down-regulation. Instead we note a lack of effect of FTY720 
alone on cytokine production in A549 cells (demonstrated in Figures 2A, 2B, 3A, 3B); thus, these data support the S1P-independence of the FTY720-mediated effects.

However, despite the fact that FTY720 did not appear to exert S1PR-mediated effects in our cellular model of airway inflammation in vitro, there remains a compelling need to develop PP2A activators that are devoid of S1P agonism. We have shown that S1P is elevated in asthma [33] and drives development of a pro-inflammatory phenotype, including IL-8 and IL-6 expression in vitro [33-35]. To this end, a chiral analog of FTY720 that does not bind S1P receptors, called $\mathrm{AAL}(\mathrm{S})$ was developed [20], and we showed that it is just as effective as FTY720 at activating PP2A complexes in vitro and significantly represses airways disease in mouse models in vivo [21]. AAL(S) is an improvement on FTY720, being devoid of S1P agonism, and we now utilize these PP2A activators in A549 lung epithelial cells to show that although they do not activate PP2A when added alone, they increased TNF $\alpha$-induced PP2A activity to inhibit epithelial cell cytokine production in a TTP-mediated manner. Further understanding of the molecular mechanism of PP2A regulation in respiratory inflammation is required, but it could be speculated that PP2A activators may repress inflammation under inflamed (mimicked here with TNF $\alpha$ ), rather than non-inflamed conditions.

Taken together our study confirms that PP2A is a druggable target in respiratory disease [21, 22] and establishes a link with TTP. Use of PP2A activators underscores the potential of enhancing TTP function with small molecules to reduce airway inflammation in respiratory disease. This new knowledge advances our understanding of the mechanistic basis of airway inflammation and suggests molecular strategies and avenues for future pharmacotherapeutic approaches based on exploiting the anti-inflammatory function of TTP. 


\section{Acknowledgements}

This research was supported by National Health and Medical Research Council of Australia APP1025637 and philanthropic funding from Mr Maurice Renshaw (Past President, Faculty of Pharmacy Foundation). NMV is supported by a Cancer Institute NSW Fellowship (10-ECF-241) and Cancer Council NSW grant (APP1030430). MMR was supported by an Endeavour Postgraduate Award and NNR received an International Postgraduate Research Scholarship.

\section{Author contributions}

AJA coordinated the study. MMR, PMH, ARC, NMV and AJA participated in research design. MMR and NNR conducted the experiments. JCM and ARC contributed reagents and analytic tools. MMR, NNR and AJA performed data analysis. MMR, NMV, ARC and AJA contributed to the writing of the manuscript. All authors reviewed the results and approved the final version of the manuscript.

\section{Conflict of interest}

The authors declare that they have no conflicts of interest. 


\section{References}

[1] A.J. Ammit, Glucocorticoid insensitivity as a source of drug targets for respiratory disease, Curr Opin Pharmacol, 13 (2013) 370-376.

[2] K.F. Chung, New treatments for severe treatment-resistant asthma: targeting the right patient, The Lancet Respiratory Medicine, 1 (2013) 639-652.

[3] P.J. Barnes, New anti-inflammatory targets for chronic obstructive pulmonary disease, Nat Rev Drug Discov, 12 (2013) 543-559.

[4] P. Prabhala, A. Ammit, Tristetraprolin and its Role in Regulation of Airway Inflammation, Mol. Pharmacol., 87 (2015) 629-638.

[5] K.R. Mahtani, M. Brook, J.L. Dean, G. Sully, J. Saklatvala, A.R. Clark, Mitogen-activated protein kinase p38 controls the expression and posttranslational modification of tristetraprolin, a regulator of tumor necrosis factor alpha mRNA stability, Mol. Cell. Biol., $21(2001)$ 6461-6469.

[6] E.A. Ross, T. Smallie, Q. Ding, J.D. O'Neil, H.E. Cunliffe, T. Tang, D.R. Rosner, I. Klevernic, N.A. Morrice, C. Monaco, A.F. Cunningham, C.D. Buckley, J. Saklatvala, J.L. Dean, A.R. Clark, Dominant Suppression of Inflammation via Targeted Mutation of the mRNA Destabilizing Protein Tristetraprolin, J. Immunol., 195 (2015) 265-276.

[7] F.P. Marchese, A. Aubareda, C. Tudor, J. Saklatvala, A.R. Clark, J.L. Dean, MAPKAP kinase 2 blocks tristetraprolin-directed mRNA decay by inhibiting CAF1 deadenylase recruitment, J. Biol. Chem., 285 (2010) 27590-27600.

[8] M. Brook, C.R. Tchen, T. Santalucia, J. McIlrath, J.S. Arthur, J. Saklatvala, A.R. Clark, Posttranslational regulation of tristetraprolin subcellular localization and protein stability 
by p38 mitogen-activated protein kinase and extracellular signal-regulated kinase pathways, Mol. Cell. Biol., 26 (2006) 2408-2418.

[9] C.R. Tchen, M. Brook, J. Saklatvala, A.R. Clark, The stability of tristetraprolin mRNA is regulated by mitogen-activated protein kinase p38 and by tristetraprolin itself, J. Biol. Chem., 279 (2004) 32393-32400.

[10] E. Hitti, T. Iakovleva, M. Brook, S. Deppenmeier, A.D. Gruber, D. Radzioch, A.R. Clark, P.J. Blackshear, A. Kotlyarov, M. Gaestel, Mitogen-activated protein kinase-activated protein kinase 2 regulates tumor necrosis factor mRNA stability and translation mainly by altering tristetraprolin expression, stability, and binding to adenine/uridine-rich element, Mol. Cell. Biol., 26 (2006) 2399-2407.

[11] T. Renda, S. Baraldo, G. Pelaia, E. Bazzan, G. Turato, A. Papi, P. Maestrelli, R. Maselli, A. Vatrella, L.M. Fabbri, R. Zuin, S.A. Marsico, M. Saetta, Increased activation of p38 MAPK in COPD, Eur. Respir. J., 31 (2008) 62-69.

[12] P. Prabhala, K. Bunge, M.M. Rahman, Q. Ge, A.R. Clark, A.J. Ammit, Temporal regulation of cytokine mRNA expression by tristetraprolin: dynamic control by p38 MAPK and MKP-1, Am J Physiol Lung Cell Mol Physiol, 308 (2015) L973-980.

[13] L. Sun, G. Stoecklin, S. Van Way, V. Hinkovska-Galcheva, R.F. Guo, P. Anderson, T.P. Shanley, Tristetraprolin (TTP)-14-3-3 complex formation protects TTP from dephosphorylation by protein phosphatase $2 \mathrm{a}$ and stabilizes tumor necrosis factor-alpha mRNA, J. Biol. Chem., 282 (2007) 3766-3777.

[14] T.P. Shanley, N. Vasi, A. Denenberg, H.R. Wong, The Serine/Threonine Phosphatase, PP2A: Endogenous Regulator of Inflammatory Cell Signaling, J. Immunol., 166 (2001) 966-972. 
[15] V. Miskolci, S. Castro-Alcaraz, P. Nguyen, A. Vancura, D. Davidson, I. Vancurova,

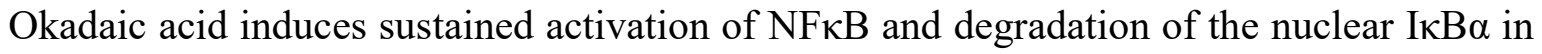
human neutrophils, Arch. Biochem. Biophys., 417 (2003) 44-52.

[16] M.R. Junttila, S.-P. Li, J. Westermarck, Phosphatase-mediated crosstalk between MAPK signaling pathways in the regulation of cell survival, FASEB J., 22 (2008) 954-965.

[17] M.M. Rahman, N.N. Rumzhum, J.C. Morris, A.R. Clark, N.M. Verrills, A.J. Ammit, Basal protein phosphatase 2A activity restrains cytokine expression: role for MAPKs and tristetraprolin, Scientific reports, 5 (2015) 10063.

[18] T.T. Cornell, V. Hinkovska-Galcheva, L. Sun, Q. Cai, M.B. Hershenson, S. Vanway, T.P. Shanley, Ceramide-dependent PP2A regulation of TNFalpha-induced IL-8 production in respiratory epithelial cells, Am. J. Physiol. Lung Cell Mol. Physiol., 296 (2009) L849-856.

[19] E.M. King, M. Kaur, W. Gong, C.F. Rider, N.S. Holden, R. Newton, Regulation of tristetraprolin expression by interleukin-1 beta and dexamethasone in human pulmonary epithelial cells: roles for nuclear factor-kappa B and p38 mitogen-activated protein kinase, J. Pharmacol. Exp. Ther., 330 (2009) 575-585.

[20] A.S. Don, C. Martinez-Lamenca, W.R. Webb, R.L. Proia, E. Roberts, H. Rosen, Essential Requirement for Sphingosine Kinase 2 in a Sphingolipid Apoptosis Pathway Activated by FTY720 Analogues, J. Biol. Chem., 282 (2007) 15833-15842.

[21] A. Collison, L. Hatchwell, N. Verrills, P.A. Wark, A.P. de Siqueira, M. Tooze, H. Carpenter, A.S. Don, J.C. Morris, N. Zimmermann, N.W. Bartlett, M.E. Rothenberg, S.L. Johnston, P.S. Foster, J. Mattes, The E3 ubiquitin ligase midline 1 promotes allergen and rhinovirus-induced asthma by inhibiting protein phosphatase 2A activity, Nat. Med., 19 (2013) 232-237. 
[22] L. Hatchwell, J. Girkin, M.D. Dun, M. Morten, N. Verrills, H.D. Toop, J.C. Morris, S.L. Johnston, P.S. Foster, A. Collison, J. Mattes, Salmeterol attenuates chemotactic responses in rhinovirus-induced exacerbation of allergic airways disease by modulating protein phosphatase 2A, J. Allergy Clin. Immunol., 133 (2014).

[23] E. Carballo, W.S. Lai, P.J. Blackshear, Feedback inhibition of macrophage tumor necrosis factor-alpha production by tristetraprolin, Science, 281 (1998) 1001-1005.

[24] T. Smallie, E.A. Ross, A.J. Ammit, H.E. Cunliffe, T. Tang, D.R. Rosner, M.L. Ridley, C.D. Buckley, J. Saklatvala, J.L. Dean, A.R. Clark, Dual-Specificity Phosphatase 1 and Tristetraprolin Cooperate To Regulate Macrophage Responses to Lipopolysaccharide, J. Immunol., 195 (2015) 277-288.

[25] Y. Amrani, A.J. Ammit, R.A. Panettieri, Jr., Tumor Necrosis Factor Receptor (TNFR) 1, but Not TNFR2, Mediates Tumor Necrosis Factor-alpha -Induced Interleukin-6 and RANTES in Human Airway Smooth Muscle Cells: Role of p38 and p42/44 Mitogen-Activated Protein Kinases, Mol. Pharmacol., 60 (2001) 646-655.

[26] S. Henness, E. van Thoor, Q. Ge, C.L. Armour, J.M. Hughes, A.J. Ammit, IL-17A acts via p38 MAPK to increase stability of TNF-alpha-induced IL-8 mRNA in human ASM, Am. J. Physiol., 290 (2006) L1283-L1290.

[27] T. Quante, Y.C. Ng, E.E. Ramsay, S. Henness, J.C. Allen, J. Parmentier, Q. Ge, A.J. Ammit, Corticosteroids reduce IL-6 in ASM cells via up-regulation of MKP-1, Am. J. Respir. Cell Mol. Biol., 39 (2008) 208-217.

[28] D. Perrotti, P. Neviani, Protein phosphatase 2A: a target for anticancer therapy, Lancet Oncol, 14 (2013) e229-e238. 
[29] K.G. Roberts, A.M. Smith, F. McDougall, H. Carpenter, M. Horan, P. Neviani, J.A. Powell, D. Thomas, M.A. Guthridge, D. Perrotti, A.T. Sim, L.K. Ashman, N.M. Verrills, Essential requirement for PP2A inhibition by the oncogenic receptor c-KIT suggests PP2A reactivation as a strategy to treat c-KIT+ cancers, Cancer Res., 70 (2010) 5438-5447.

[30] I. Cristobal, R. Manso, R. Rincon, C. Carames, C. Senin, A. Borrero, J. Martinez-Useros, M. Rodriguez, S. Zazo, O. Martinez-Aguilera, J. Madoz-Gurpide, F. Rojo, J. GarciaFoncillas, PP2A inhibition is a common event in colorectal cancer and its restoration using FTY720 shows promising therapeutic potential, Molecular Cancer Therapeutics, DOI 10.1158/1535-7163.mct-13-0150(2014).

[31] Y. Kobayashi, N. Mercado, P.J. Barnes, K. Ito, Defects of protein phosphatase 2A causes corticosteroid insensitivity in severe asthma, PLoS One, 6 (2011) e27627.

[32] Y. Kobayashi, N. Mercado, A. Miller-Larsson, P.J. Barnes, K. Ito, Increased corticosteroid sensitivity by a long acting beta2 agonist formoterol via beta2 adrenoceptor independent protein phosphatase 2A activation, Pulm. Pharmacol. Ther., 25 (2012) 201-207.

[33] A.J. Ammit, A.T. Hastie, L.C. Edsall, R.K. Hoffman, Y. Amrani, V.P. Krymskaya, S.A. Kane, S.P. Peters, R.B. Penn, S. Spiegel, R.A. Panettieri, Jr., Sphingosine 1-phosphate modulates human airway smooth muscle cell functions that promote inflammation and airway remodeling in asthma, FASEB J., 15 (2001) 1212-1214.

[34] M.M. Rahman, H. Alkhouri, F. Tang, W. Che, Q. Ge, A.J. Ammit, Sphingosine 1-phosphate induces neutrophil chemoattractant IL-8: repression by steroids, PLoS One, 9 (2014) e92466.

[35] W. Che, J. Parmentier, P. Seidel, M. Manetsch, E.E. Ramsay, H. Alkhouri, Q. Ge, C.L. Armour, A.J. Ammit, Corticosteroids Inhibit S1P-Induced IL-6 Secretion From Human 
Airway Smooth Muscle via MKP-1-Mediated Repression of MSK1, Am. J. Respir. Cell Mol. Biol., 50 (2014) 358-368. 


\section{Figure Legends}

Figure 1. PP2A activators, FTY720 and AAL(S), enhance TNFa-induced PP2A phosphatase activity in A549 cells. A549 cells were pretreated with (A) $2.5 \mu \mathrm{M}$ FTY720 or (B) $2.5 \mu \mathrm{M}$ AAL(S) for $6 \mathrm{~h}$, compared to vehicle-treated controls. Cells were treated with vehicle or $\mathrm{TNF} \alpha(4 \mathrm{ng} / \mathrm{ml})$ and then PP2A enzymatic activity (measured as pmol free phosphate) detected at $1 \mathrm{~h}$. Statistical analysis was performed using one-way ANOVA then Bonferroni's post-test (where * denotes a significant effect of TNF $\alpha$ compared to vehicle-treated cells, and $\S$ denotes a significant effect of PP2A activators on TNF $\alpha$-induced PP2A activity $(P<0.05))$. Data are mean+SEM values from $n=3$ independent experiments.

Figure 2. PP2A activators, FTY720 and AAL(S), significantly repress TNFa-induced IL-8 mRNA expression and protein secretion. A549 cells were pretreated with (A, B) $2.5 \mu \mathrm{M}$ FTY720 or (C, D) $2.5 \mu \mathrm{M}$ AAL(S) for $6 \mathrm{~h}$, compared to vehicle-treated controls. Cells were treated with vehicle or $\mathrm{TNF} \alpha(4 \mathrm{ng} / \mathrm{ml})$ and then (A, C) IL-8 mRNA expression (results expressed as fold increase compared to vehicle-treated cells at $0 \mathrm{~h}$ ) and (B, D) IL-8 protein secretion measured (results expressed as a percentage of TNF $\alpha$-induced IL-8 secretion at $24 \mathrm{~h}$ : i.e. B) $51,670.5 \pm 2,465.2 \mathrm{pg} / \mathrm{ml}$ and D) $48,825.4 \pm 2492.5 \mathrm{pg} / \mathrm{ml}$ ) at the indicated times. Statistical analysis was performed using two-way ANOVA then Bonferroni's post-test (where * denotes a significant effect of TNF $\alpha$ compared to vehicle-treated cells, and $\S$ denotes significant repression of TNF $\alpha$-induced cytokine production by PP2A activators $(P<0.05))$. Data are mean+SEM values from $n=4-5$ independent experiments. 
Figure 3. PP2A activators, FTY720 and AAL(S), significantly repress TNFo-induced IL-6 mRNA expression and protein secretion. A549 cells were pretreated with (A, B) $2.5 \mu \mathrm{M}$ FTY720 or (C, D) $2.5 \mu \mathrm{M}$ AAL(S) for $6 \mathrm{~h}$, compared to vehicle-treated controls. Cells were treated with vehicle or TNFa $(4 \mathrm{ng} / \mathrm{ml})$ and then (A, C) IL-6 mRNA expression (results expressed as fold increase compared to vehicle-treated cells at $0 \mathrm{~h}$ ) and (B, D) IL-6 protein secretion measured (results expressed as a percentage of TNF $\alpha$-induced IL-6 secretion at 24 h: i.e. B) $1,742.6 \pm 274.7 \mathrm{pg} / \mathrm{ml}$ and D) $1650.3 \pm 192.4 \mathrm{pg} / \mathrm{ml}$ ) at the indicated times. Statistical analysis was performed using two-way ANOVA then Bonferroni's post-test (where * denotes a significant effect of TNF $\alpha$ compared to vehicle-treated cells, and $\S$ denotes significant repression of TNF $\alpha$-induced cytokine production by PP2A activators $(P<0.05))$. Data are mean+SEM values from $n=4-5$ independent experiments.

Figure 4. Specific knockdown of TTP with siRNA increases TNFa-induced IL-8 and IL-6 mRNA expression and protein secretion. A549 cells were either left untransfected or transfected with scrambled control (scr) or siRNA against TTP (siRNA), then treated with vehicle or TNFa (4 ng/ml) before: (A, B) knockdown of TTP mRNA and TTP protein expression confirmed at $1 \mathrm{~h}$ by (A) RT-PCR and (B) Western blotting (compared to $\alpha$-tubulin as loading control); (C, E) IL-8 and IL-6 mRNA expression measured at $1 \mathrm{~h}$ (results expressed as fold increase compared to untransfected vehicle-treated controls); and (D, F) IL-8 and IL-6 protein secretion measured at $24 \mathrm{~h}$. Statistical analysis was performed using Student's unpaired $t$ test (where * denotes a significant effect of TNF $\alpha$ compared to untransfected vehicle-treated controls, and $\S$ denotes a significant effect of siRNA against TTP compared to untransfected 
controls treated with TNF $\alpha(P<0.05))$. Data are mean+SEM values from $\mathrm{n}=3$ independent experiments.

Figure 5. PP2A activators do not enhance TTP mRNA expression; rather they modify TTP's dynamic equilibrium. (A, B) A549 cells were pretreated with (A) $2.5 \mu$ M FTY720 or (B) $2.5 \mu \mathrm{M}$ AAL(S) for $6 \mathrm{~h}$, compared to vehicle-treated controls. Cells were treated with vehicle or TNF $\alpha(4 \mathrm{ng} / \mathrm{ml})$ and then TTP mRNA expression measured (results expressed as fold increase compared to vehicle-treated cells at $0 \mathrm{~h}$ ) at the indicated times. Statistical analysis was performed using two-way ANOVA then Bonferroni's post-test (where * denotes a significant effect of TNF $\alpha$ compared to vehicle-treated cells $(P<0.05))$. Data are mean+SEM values from $\mathrm{n}=4-5$ independent experiments. (C-F) A549 cells were treated with: (C) TNFa (4 ng/ml) for 0, 1, 2, 4, 8, 24 h; (D, E, F) pretreated with vehicle, $2.5 \mu \mathrm{M}$ FTY720 or $2.5 \mu \mathrm{M}$ AAL(S) for $6 \mathrm{~h}$, then treated with vehicle or TNF $\alpha(4 \mathrm{ng} / \mathrm{ml})$ for $1 \mathrm{~h}, 2 \mathrm{~h}$ or $0.25 \mathrm{~h}$, respectively. Western blotting for TTP was performed (compared to $\alpha$-tubulin as loading control). Please note two bands of immunoreactivity for TTP: bands at higher molecular weight indicate stable phosphorylated TTP (inactive), while lower bands are unphosphorylated (active) but unstable. Results are representative Western blots or densitometric analysis (normalized to $\alpha$-tubulin and expressed as $\%$ TNF $\alpha$-induced TTP protein upregulation at either $1 \mathrm{~h}(\mathrm{D})$ or $2 \mathrm{~h}(\mathrm{E}))$. Statistical analysis was performed using one-way ANOVA then Bonferroni's post-test (where * denotes a significant effect of TNF $\alpha$, and $\S$ denotes a significant effect of PP2A activators on TNF $\alpha$-induced effects $(P<0.05))$. Data are mean+SEM values from $n=3-4$ independent experiments. $(\mathrm{F})$ Results for $\mathrm{p} 38$ MAPK phosphorylation (compared to total p38 MAPK as a loading control) are representative Western blots of $n=3$ independent experiments. 
Figure 6. Specific knockdown of TTP with siRNA reverses repression of TNF $\alpha$-induced IL8 and IL-6 mRNA expression and protein secretion by the PP2A activator FTY720. A549 cells were either left untransfected or transfected with scrambled control (scr) or siRNA against PP2A-C, then pretreated for $6 \mathrm{~h}$ with vehicle or with $2.5 \mu \mathrm{M}$ of FTY720. Cells were stimulated with TNF $\alpha$ (4 ng/ml) before: (A) knockdown of TTP mRNA expression confirmed or (B, D) IL8 and IL-6 mRNA expression measured at $1 \mathrm{~h}$ (results expressed as a percentage of TNF $\alpha$ induced mRNA expression in untransfected controls); (C, E) IL-8 and IL-6 protein secretion measured at $24 \mathrm{~h}$ (results expressed as a percentage of $\mathrm{TNF} \alpha$-induced protein secretion in untransfected controls). Statistical analysis was performed using Student's unpaired $t$ test (where: * denotes significant effect of TTP against siRNA and $\S$ significant repression of TNF $\alpha$-induced cytokine production by FTY720 (compared to untransfected controls); $\infty$ denotes that specific knockdown of TTP with siRNA reverses repression of TNF $\alpha$-induced IL-8 and IL-6 mRNA expression and protein secretion by FTY720 (compared to untransfected controls) $(P<0.05)$. Data are mean+SEM values from $n=3$ independent experiments. 